\title{
Surface Contouring with a Range Finder
}

\author{
Thomas J. Todd*
}

Arlene Lennox

August, 1986

*Present address: Kaneland High School

47W326 Keslinger Road 


\title{
Surfece Contouring With Range Finder
}

\author{
by Thomas J. Todd*
} Arlene Lennox

August, 1986

\begin{abstract}
:
New patients at the Neutron Therapy Facility must be contoured so that the staff might offer safe and effective treatment. A new system using the lasers and rotating chair that are in the treatment room, a range finder that projects a scale, a contour board marked with polar coordinates, and a swing scale has been implemented. This system significantly improves the accuracy of the contours drawn and should prove to be a time saver in difficult contours, such as those found about the head. Patients will benefit in that the contouring process will be more efficient and accurate.

This system may be of use in other situations at Fermilab needing surface contouring through a cross section. Its scale is marked in whole units, and requires low background light conditions to be seen. Modification of the geometry of NTF's system can change the spacing of marks on the scale, but readings would no longer be in $1 \mathrm{~cm}$ units, and would require a conversion factor to draw a contour at full scale. The range finder is accurate at distances less than $55 \mathrm{~cm}$ from the isocenter.§
\end{abstract}

Our thanks to Morris Tatcher of Rambam originally suggested the use of the range finder as a contouring device. 


\section{Contouring Equipment}

\section{Parts of the system}

it is helpful at this point to become familiar with the tools involved in the use of the range finder contouring system. Sketches of each tool can be found below for easy identification.

The range finder itself is composed of a light barrel and a wall mount. The light barrel contains a 15 watt bulb, a scale lens, and a focusing lens. Each is held securely in place by screws that are imbedded in the barrel and are turned with the use of a .050 Allen wrench. If for any reason the barrel must be removed from the wall mount, consult the "Range Finder" information in the System Dimensions section of this manual. It is very important that the barrel be returned to exactly the same position it had before it was removed. The wall mount locks the position of the barrel. There are three knobs on the wall mount. The knob that is located on top of the cylinder part of the wall mount holds the barrel in place. This knob should be tightened firmly so that the barrel is held without undo pressure. If the knob is tightened too much, a distortion of the shape and the orientation of the barrel may result. This in turn would reduce the accuracy of the system. The other knobs prevent the cylinder that holds the barrel from tilting vertically.

The treatment chair is located upon an elevator. The chair is designed to rotate on a vertical axis about the iso-center of the treatment room. The base of the chair is marked at 10 degree increments with two scales, $X$ and $N$. All contouring readings are done on the $N$ scale as read from a monitor, and then converted to $X$ scale for plotting. It is possible to rotate the chair through as small as 1 degree steps.

The contour board is marked radially at 10 degree intervals, with multiples of thirty having bolder lines. The numbers at the outer ends of these lines are $X$ values of the chair, arranged in such a way as to adjust for the differences in the orientation of the chair and the position of the range finder, and for the manipulation of the contour that will present a standard medical view of the cross-section (distal view, anterior on top). At the center of the contour board is a metal post and clasp. These are designed to hold the swing scale in place while contouring is in progress.

The swing scale is a ruler-like device. It has a tab that attaches the scale to the post of the contour board. The numbers on the swing scale correspond with the numbers on the scale projected by the
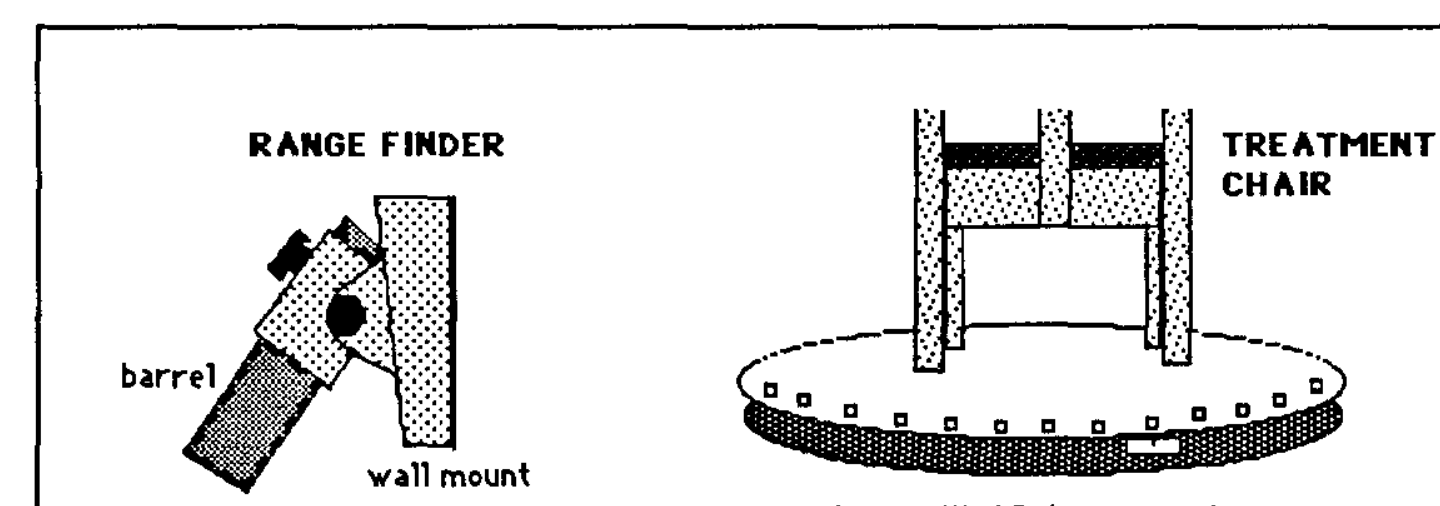

base with 10 degree marks

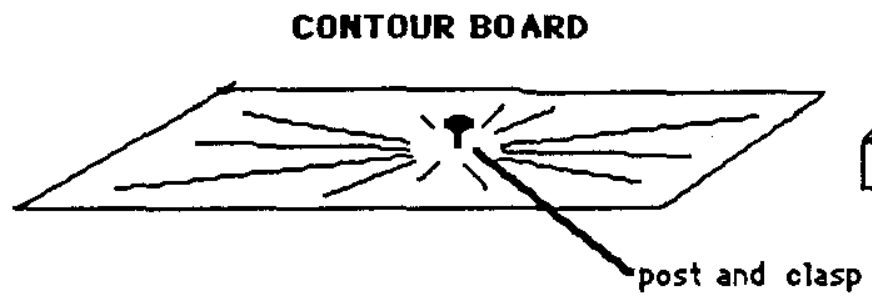

SYING SCALE

tab

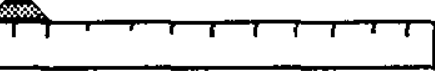




\section{Contouring Session}

\section{Beadine the value at the iso-center}

Before beginning a series of contours, one must read the value of the range finder scale at the iso-center. This reading will determine where the swing scale's tab must be punctured by the post of the contour board. This value is fairly easy to obtain.

When the laser strikes a surface, it traces a line across it. The treatment room has three sets of lasers that all meet at the iso-center (see figure at top right). A surface that would contain the iso-center would have all three trace lines crossing at its' location. By holding a piece of paper or an index card on the iso-center, it is possible to obtain a reading from the range finder scale (see figure at bottom right).

The following process is used in locating the iso-center on a paper or card. Hold the card so that it faces the corner of the treatment room between the $x$-ray source and the range finder, and at an angle to the floor. The iso-center is located over the treatment chair. Move the card in this area until you see three laser traces that form a triangle. Experiment with small adjustments in the position of the card, trying to shrink the size of the triangle until the lines all cross at one point. Read the range finder scale at this point, trying for an accuracy of at least half of a centimeter.

If the range finder is left undistrubed, this iso-center value should remain constant. The value achieved for the Fermi treatment room has been $125.0 \mathrm{~cm}+/$ $0.1 \mathrm{~cm}$. $\S$

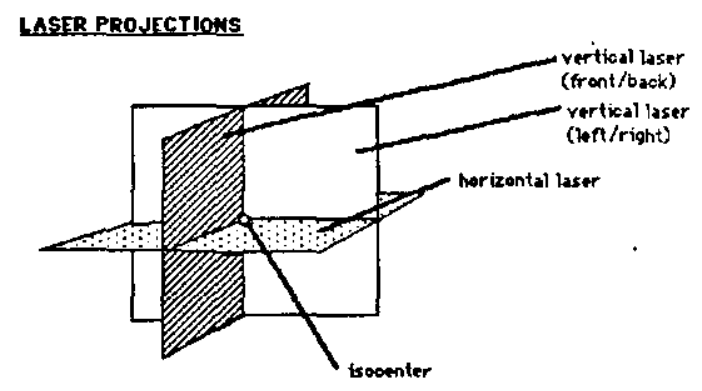

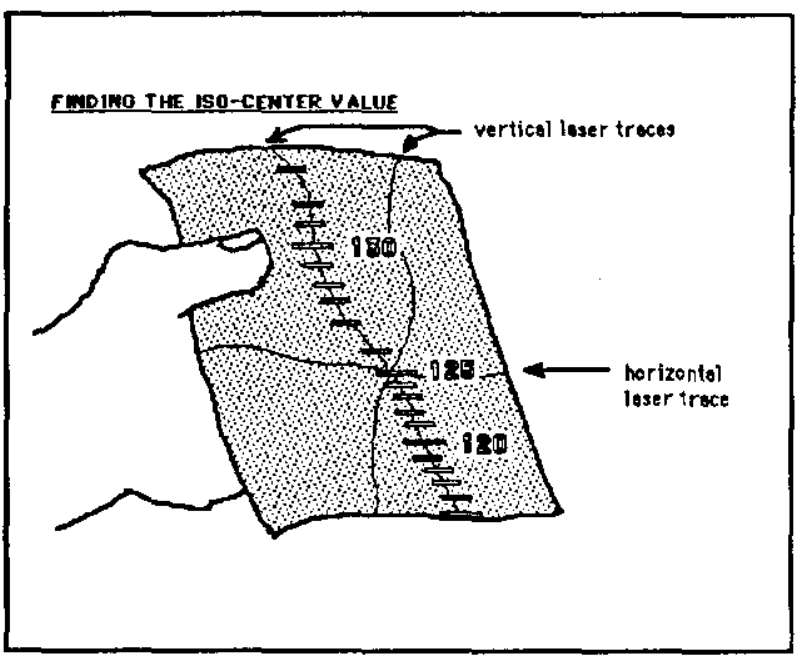

\section{Chair orientation and elevation}

The usual position of the treatment chair is facing "front", that is, at an $N$ reading of 0 degrees. The contouring process could be started at any $N$ reading, though a multiple of 10 is preferred. Make certain that the chair is at the locked position on the track of the elevator's floor. The patient is now placed in the chair and secured. The position of the patient must not change with respect to the iso-center. Lower the elevator until the trace of the horizontal laser on the patient is at the desired level for the contour. Using the vertical lasers, try to locate the iso-center as near the center of the cross-section as possible. This will give range finder values over a more narrow range of numbers, increasing the accuracy and centering the contour on the rectangular grid paper.

Do not release the patient or change the elevator position until you are satisfied that the contour is accurate and complete. If the elevator or patient should move before contouring is finished, the location of the isocenter in the patient will have changed. It would be necessary to start over. $\S$ 


\section{Plotting contour points}

Two technicians are needed to contour a patient. One technician operates the elevator and chair. The other takes readings from the surface of the patient and plots them on rectangular grid paper placed on the contour board. Readings are first recorded to a chart (see last page) as $N$ values, and then plotted from the $X$ values on the chart to the grid paper on the contour board.

Anyone who has worked a "dot-to-dot" puzzle knows that usually the mysterious shape reveals itself before all the points have been connected. This often leads to finishing the drawing without reading the last few numbers. This contouring system operates on that principle. The person plotting contour readings and sketching the contour has a good idea of the shape of the cross-section. The points are there only to control the size and proportion of the contour.

It is highly unlikely that straight line connections between points would give the actual shape of the patient. It is helpful to have some experience at curve drawing through a set of data points. The closeness of the points on the grid paper will depend largely upon the detail required of the surface and the drawing ability of the technician.

\section{Step 1}

Place the rectangular grid paper on the contour board so that you can see the readings around the edge of the board. Punch the post through the grid paper and the tab on the swing scale at the iso-center reading. Tape two opposite corners of the grid paper to the contour board and put the clasp on top of the post.

\section{Step 2}

Align the swing scale along the chair's $N$ value reading. Read the value of the range finder scale where the laser traces intersect on the surface of the patient. Try to estimate as closely as you can the actual value at the surface. Place a card on this spot if the surface cannot be read easily due to curvature or hair.

\section{Step 3}

Record on the chart the scale reading at that $\mathrm{N}$ position. Find the $X$ value reading that corresponds with the $\mathrm{N}$. Plot the point using the swing scale. The chair operator turns the chair to the new $\mathrm{N}$ reading.

Repeat steps 2 and 3 untll the grid has

\section{Step 4}

Examine the contour points before drawing curves. Look for points that seem out of place. If necessary, tum the chair back to that reading and read the range finder scale again. Make sure to plot the fractional part of the reading on the correct side of the whole number.

Get additional readings if an area needs more points to accurately contour.

\section{Step 5}

Quickly sketch in the curves between points. Check the dimensions of your contour drawing with those of the patient using calipers. Two caliper readings (left-right and front-back) should be sufficient to check size accuracy.

A difference of half a centimeter or less between the contour drawing and the patient is acceptable.

\section{Step 6}

If the difference between the caliper and contour drawing dimensions is too great, follow the process in step 4 to verify the accuracy of your points.

When the contour drawn is acceptable, the patient may be released and the elevator position changed.

\section{$\S$}

\section{Becommended Dearee Intervals}

5 - high detail areas, ie. nose
10 - general detail, ie. ear

20 - low detail, ie. back 


\section{System Dimensions}

\section{The range finder}

Perhaps the most important tool of the entire system is the range finder. The NTF's range finder has the dimensions shown in the figure below. The barrel would have to be removed from the mount in order to replace a light bulb. The light barrel and mount have been marked so that the unit may be reassembled to identical dimensions. The cylinder of the wall mount has also been marked to insure the preservation of the barrel angle.

The range finder draws 3 amps on a 6 volt transformer. The 15 watt bulb is accessible from the rear of the barrel. To replace a bulb, loosen the knob located on top of the wall mount's cylinder and remove the barrel. At the rear of the light barrel is the bulb assembly. The bulb assembly is removed by turning a small embedded bolt at the rear of the barrel with a .050 Allen wrench. The barrel may then be separated from the light assembly. The bulb is similar to those often found in automobiles, and may be removed by pushing the bulb down into the light assembly and turning. After the new bulb is inserted, place the light assembly back in the barrel. Make sure the bolt on the barrel is in line with the groove of the light assembly. Tighten the bolt, return the barrel to its proper position in the wall mount's cylinder, adjust the cylinder to obtain the desired angle, and check the scale reading at the isocenter.
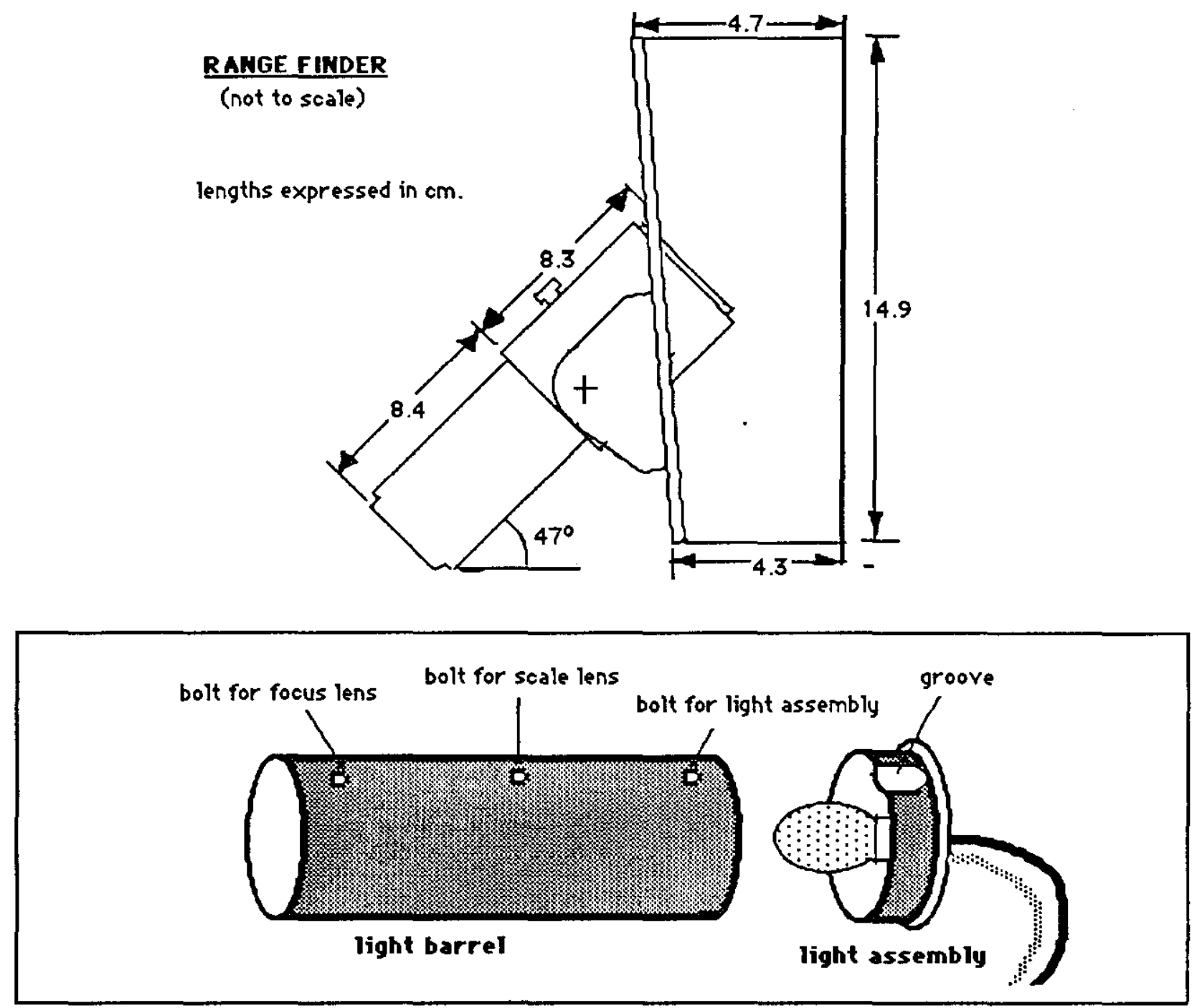


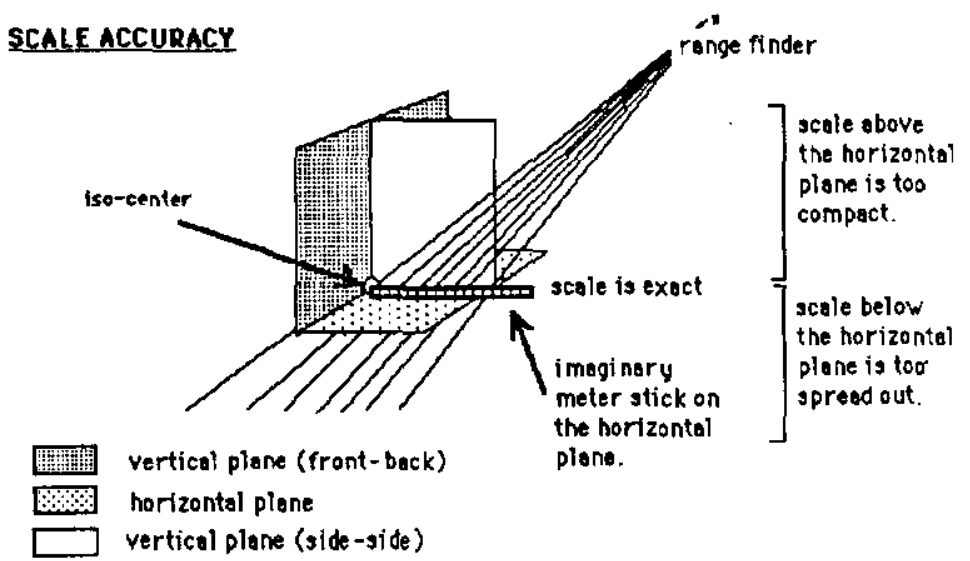

The range finder system for contouring has a delicate geometry. The nature of the scale projected by the range finder is sensitive to the height above the iso-center and the angle with respect to the horizontal plane.

If the scale is projected toward the horizontal plane at an angle that is not $47^{\circ}$, the result is an uneven spacing of the marks. This in turn produces a significant narrowing of the range over which the scale may be used. The height of the scale above the iso-center also affects the spacing of each mark, even if the angle was correct. At $76.0 \mathrm{~cm}$ above the level of the isocenter, NTF's range finder projects a fairty wide band of marks at the desired $1 \mathrm{~cm}$ interval. It was found, however, that the numerical readings on this scale were not the

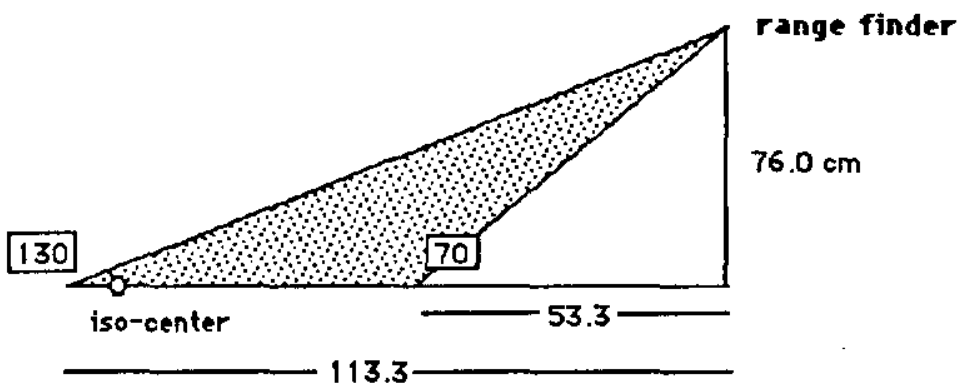
actual horizontal distance from the iso-center to the plane of the range finder.

The most accurate spacings occurred from readings of 70 through 130 . The 70 reading was actually at a distance of $53.3 \mathrm{~cm}$ from the vertical plane of the range finder. (see figure above) This was not significant, however, since all measurements are taken with respect to the iso-center. As long as the iso-center falls within the range of accurate markings, simple subtraction will yield radius values. This subtraction step was avoided at NTF by marking a centimeter ruler with the values that matched those in the accurate zone of the range finder. By locating the post of the contour board at the iso-center value, data points can be located by simply plotting the range finder reading using the swing scale. $\S$ 